\title{
SeArChing For TEV DARK MATter By ATMOSPheric ČERENKOV TECHNIQUES
}

\author{
M. Urban* \\ A. Bouquet ${ }^{\dagger}$ \\ B.Degrange* \\ P. Fleury* \\ J. Kaplan ${ }^{\dagger}$ \\ A.L. Melchior ${ }^{\dagger}$ and E. Paré*
}

\begin{abstract}
There is a growing interest in the possibility that dark matter could be formed of weakly interacting particles with a mass in the $100 \mathrm{GeV}-2 \mathrm{TeV}$ range, and supersymmetric particles are favorite candidates. If they constitute the dark halo of our Galaxy, their mutual annihilations produce energetic gamma rays that could be detected using existing atmospheric Cerenkov techniques.
\end{abstract}

To appear in Physics Letters B July 1992

${ }^{*}$ Laboratoire de Physique Nucléaire et Hautes Energies, Ecole Polytechnique/CNRS-IN2P3, 91128 Palaiseau Cedex , FRANCE

${ }^{\dagger}$ LPTHE Universités PARIS 6 and 7, Unité associée au CNRS UA 280, 2 Place Jussieu, 75251 Paris Cedex 05, FRANCE 


\section{Introduction}

Galaxies seem embedded in massive spheroidal dark halos [1, 2, 3, 代. Extensions of the standard model of particle physics often predict the existence of a new stable particle, a natural candidate for halo dark matter. The residual abundance of a particle after the Big-Bang depends on its mass and interactions, and to account for the dark matter puzzle a weakly interacting massive particle (W.I.M.P.) is needed, for instance a neutrino-like particle with a mass around $1 \mathrm{TeV}$. Such particles will be very difficult to detect, even by accelerator experiments (LHC-SSC) of next century. Halo particles can be directly detected when they cross a bolometer detector [5, 6] but the flux is low above $50 \mathrm{GeV}$. They can also be indirectly detected through their annihilation products [7, 8, 9, 10, 11, 12] : annihilations after their capture in the Earth or in the Sun producing neutrinos, or annihilations in the halo producing energetic cosmic rays (gamma rays, electrons, protons, antiprotons...). Existing models for our galactic halo predict a strong annihilation rate near the center of the Galaxy. We focus here on the annihilations into photons, because photons conserve their initial direction. Each annihilation produces either primary photons (leading to a gamma line signal) or secondary photons from the decay of primary annihilation products. The fluxes are low again for a large WIMP mass, but this can be balanced by the large acceptance of detectors using the atmospheric Cerenkov technique like the existing Atmospheric Črenkov Telescope (ACT) of the Whipple Observatory [13]. With similar instruments, it should be possible to detect for instance the leading WIMP candidate, the neutralino predicted by supersymmetry [14].

\section{ACT's and backgrounds}

The ACT detects incoming cosmic rays through the Cerenkov light emitted by the shower of secondary particles created when the cosmic ray (photon, electron, proton, heavier nucleus...) collides with a nucleus in the upper atmosphere [13]. The image shape analysis affords an efficient gamma versus hadron separation. The Čerenkov light covers a disk of about $120 \mathrm{~m}$ radius on the Earth surface (an area of 45000

$\mathrm{m}^{2}$ ), and the detector must be somewhere in this area to see the cosmic ray shower. Less energetic cosmic rays produce less Čerenkov light, and the minimal detectable energy (threshold) is linked to the size of the mirrors which collect this light. The best observation conditions correspond to a cosmic ray within 45 degrees from the zenith. As we shall see, the photon flux from WIMP annihilations is largest from the galactic center, which can be seen at zenith only if the observatory is built in the southern hemisphere, at a latitude near -29 degrees. Observation of Cerenkov light is only possible at night when there is no moon, and so the galactic center can be observed for $t_{o b s} \simeq 2 \times 10^{6} \mathrm{~s}$ per year. The effective angular acceptance $\Delta \Omega$ of present ACT's is about $10^{-3}$ sr with an angular resolution of $3 \times 10^{-6}$ sr, so that they are well suited to the study of localized sources. The usual procedure consists in the comparison of the flux from an expected source with neighbouring sky regions (on-off experiment).

To be definite, we shall use as a reference ACT the Granite set-up at the Whipple Observatory, which uses two mirrors of about $10 \mathrm{~m}$ diameter, $120 \mathrm{~m}$ apart, leading at present to a minimal detectable energy around $200 \mathrm{GeV}$. Increasing the number and/or the size of mirrors may lower this threshold. The two telescopes effectively survey $63000 \mathrm{~m}^{2}$ if they are used independantly, but their effective detection area $S$ is only $18000 \mathrm{~m}^{2}$ when the two telescopes are used in coincidence to give the best energy resolution and an increased hadronic rejection. The energy of a gamma cosmic ray is determined from a stereoscopic view with a precision $\sigma(E) / E \simeq 0.1$. Adding other telescopes, about $120 \mathrm{~m}$ apart, would increase the effective area and therefore lower the detectable cosmic flux. The product $S \Delta \Omega t_{o b s}$ is a measure of the 
efficiency of the telescope to detect cosmic rays, and $S \Delta \Omega t_{o b s}=3.6 \times 10^{11} \mathrm{~cm}^{2} \mathrm{sr}$ s for our reference ACT.

There is a large background to the WIMP annihilation signal that we are looking for. Cosmic ray hadrons must be rejected, and experience at the Whipple Observatory shows that a rejection factor of $10^{3}$ should be reached for two detectors in coincidence. The flux of misidentified hadrons then follows a power law [13]:

$$
\frac{\mathrm{d} N_{h}}{\mathrm{~d} t \mathrm{~d} S \mathrm{~d} \Omega \mathrm{d} E}=6 \times 10^{-9}\left(\frac{E}{1 \mathrm{TeV}}\right)^{-2.75} \text { photon - like hadrons }\left(\mathrm{s} \mathrm{cm}^{2} \mathrm{sr} \mathrm{TeV}\right)^{-1}
$$

When a hadron is misidentified as a photon, its energy is underestimated, and the preceding formula takes this effect into account. Electrons cannot be distinguished from photons with atmospheric Cerenkov detectors, as their showers have the same development as photons in the atmosphere. The electron flux is measured at energies up to $1 \mathrm{TeV}$ [15, 16]. The spectrum also follows a power law :

$$
\frac{\mathrm{d} N_{e}}{\mathrm{~d} t \mathrm{~d} S \mathrm{~d} \Omega \mathrm{d} E}=6 \times 10^{-9}\left(\frac{E}{1 \mathrm{TeV}}\right)^{-3.3} \text { electrons }\left(\mathrm{s} \mathrm{cm}^{2} \mathrm{sr} \mathrm{TeV}\right)^{-1}
$$

The electron background dominates the background of misidentified hadrons up to $1 \mathrm{TeV}$. The fluxes of electrons or hadrons are isotropic. Finally, there is the diffuse photon background itself, supposed to be due to the collisions of cosmic ray protons on interstellar gas. The spectrum from the galactic center was measured by the COS-B satellite [17, 18, 19] up to $5 \mathrm{GeV}$ :

$$
\frac{\mathrm{d} N_{\gamma}}{\mathrm{d} t \mathrm{~d} S \mathrm{~d} \Omega \mathrm{d} E}=0.35 \times 10^{-9}\left(\frac{E}{1 \mathrm{TeV}}\right)^{-2.75} \text { photons }\left(\mathrm{s} \mathrm{cm}^{2} \mathrm{sr} \mathrm{TeV}\right)^{-1}
$$

This power law is the same as for the hadron spectrum, and we may assume that Equation 3 extends to higher energies. This is not in disagreement with upper bounds on the photon flux above $5 \mathrm{GeV}$ [15, 16]. This flux is smaller than the electron background.

A signal can be detected even if it is small compared to the background, provided that the background is measured with a good precision. In our case, an on source-off source method will be available, allowing for a reliable estimation of this background. The noise is the uncertainty on the background, that is the square root of the number $N_{\text {backgr }}$ of all photon-like events (including electrons and misidentified hadrons) detected during the experiment time $t_{o b s}$ in a given energy bin $\Delta E$, that we shall take as $3 \sigma(E) \simeq 0.3 E$. For a detector of acceptance $S \Delta \Omega$, this number is :

$$
N_{\text {backgr }}=S \Delta \Omega t_{\text {obs }}\left\{\frac{\mathrm{d} N_{\gamma}}{\mathrm{d} t \mathrm{~d} S \mathrm{~d} \Omega \mathrm{d} E}+\frac{\mathrm{d} N_{e}}{\mathrm{~d} t \mathrm{~d} S \mathrm{~d} \Omega \mathrm{d} E}+\frac{\mathrm{d} N_{h}}{\mathrm{~d} t \mathrm{~d} S \mathrm{~d} \Omega \mathrm{d} E}\right\} \Delta E
$$

We must now compare these numbers to the expected signal flux from halo WIMP annihilations.

\section{WIMP annihilation from the galactic center}

We assume that our Galaxy is embedded in a dark halo made of WIMP's of mass $m_{\chi}$ which annihilate in pairs. The annihilation rate is $\left\langle\sigma V>_{\text {halo }} n_{\chi}^{2}\right.$, where $\left\langle\sigma V>_{\text {halo }}\right.$ is the (thermal average in the halo of the) annihilation cross-section $\sigma$ times the relative WIMP velocity $V, n_{\chi}=\rho_{\chi} / m_{\chi}$ is the dark matter number density. At large distance $r$ from the center, the halo density decreases as :

$$
\rho(r) \simeq \rho_{\odot} \frac{a^{2}+r_{\odot}^{2}}{a^{2}+r^{2}}
$$


where $\rho_{\odot}=0.3 \pm 0.1 \mathrm{GeV} / \mathrm{cm}^{3}$ is the dark matter density in the solar neighbourhood, $a=5 \pm 3 \mathrm{kpc}$ is the core radius and $r_{\odot}=8.5 \mathrm{kpc}$ is the distance of the Sun to the galactic center [20, 21, 22, 23. The annihilation rate of this dark matter far away from the galactic center turns out to be too low to be detected with present size ACT's.

The dark matter density may however be much larger at the center, as there seems to be a massive nucleus at the center of the Galaxy [24, 25. According to the isothermal model of Ipser and Sikivie [26], this nucleus induces an enhancement of the dark matter density within a $150 \mathrm{pc}$ radius around the center, with a density at center of :

$$
\rho \simeq 0.34 \alpha \mathrm{TeV} / \mathrm{cm}^{3}
$$

The $\alpha$ parameter $(0.1<\alpha<3)$ reflects the uncertainties on the rotation velocity of the galaxy, and on the contribution of luminous matter to this velocity. Annihilation photons in the TeV energy range are unlikely to be absorbed by baryonic matter in the galactic center since, in the worst case of a completely gaseous core, a $\gamma$-ray crosses less than one radiation length. As seen from Earth, the central region covers $\Delta \Omega=10^{-3}$ sr, nicely corresponding to the acceptance of present ACT's. We can perform an on-off experiment, aiming at the galactic center and comparing with the result off center. The $10^{-6} \mathrm{sr}$ ACT angular resolution is small enough to distinguish point sources inside these $10^{-3}$ sr from the more diffuse WIMP annihilation cloud.

Photons appear in the decay chain of the primary annihilation products (quarks, leptons, Higgs scalars, $\mathrm{W}^{ \pm}$or $\mathrm{Z}^{0}$ 's, etc.), and their energy is then continuously distributed. Their distribution $\mathrm{d} n_{\gamma}(E) / \mathrm{d} E$ can be computed through a Lund Monte-Carlo 27] (Figure 1), and depends only weakly on the WIMP model. The integral $\int \mathrm{d} n_{\gamma}(E)$ is the photon multiplicity per annihilation. The number of photons due to WIMP annihilations within $150 \mathrm{pc}$ from the center, and received on our reference telescope is :

$$
N_{\gamma}(E) \mathrm{d} E=S t_{o b s} \mathrm{~d} n_{\gamma}(E) \frac{<\sigma V>_{\text {halo }}}{4 \pi r_{\odot}^{2}} \int_{0}^{150 \mathrm{pc}}\left(\frac{\rho(r)}{m_{\chi}}\right)^{2} 4 \pi r^{2} \mathrm{~d} r
$$

WIMP annihilations outside the $150 \mathrm{pc}$ sphere, but inside the $10^{-3}$ sr cone, do not contribute to a signal defined by an on-source minus off-source subtraction. The cross-sections $\sigma$ and the corresponding energy spectra $\mathrm{d} n_{\gamma}(E) / \mathrm{d} E$, reduced to a delta function in the case of monochromatic photons, are summed over all relevant annihilation channels.

Two different signals can be looked for at the same time. We can look for a gamma line due to the direct annihilation of WIMP's into photons, or we can look for the total excess of soft photons due to the decays of all annihilation products.

\section{Soft photons}

The Lund Monte-Carlo codes [27] typically indicate that 50 photons or so are produced in the decay chains, but that most of them are at a very low energy compared to the WIMP mass $m_{\chi}$ (see Figure 1). The signal is the number of photons received above the ACT energy threshold $E_{\text {thresh }}$. Table 1 shows the integral $\int \mathrm{d} n_{\gamma}(E)$ from $E_{\text {thresh }}$ to $m_{\chi}$ as a function of $m_{\chi}$ for a quark-antiquark channel (the result being very similar for other channels).

\begin{tabular}{|l|r|r|r|r|r|}
\hline$m_{\chi}(\mathrm{TeV})$ & 0.4 & 0.8 & 1.0 & 2.0 & 4.0 \\
\hline$E_{\text {thresh }}=0.1 \mathrm{TeV}$ & 0.02 & 0.17 & 0.32 & 1.45 & 4.40 \\
\hline$E_{\text {thresh }}=0.2, \mathrm{TeV}$ & 0.0004 & 0.02 & 0.05 & 0.35 & 1.55 \\
\hline
\end{tabular}


Table 1 : Number of soft decay photons above threshold. The first line gives the mass $m_{\chi}$ of the particles which annihilate into a quark-antiquark pair, the second line gives the number of decay photons with an energy above $0.1 \mathrm{TeV}$, and the third line the number of photons above $0.2 \mathrm{TeV}$.

Note the great sensitivity to the threshold : the number of photons above the treshold increases roughly as $m_{\chi}^{2}$, and, as a result, the signal can only be detected if the WIMP mass is far above threshold. From Equation 7, and for our reference telescope, this signal is :

$$
\text { Soft photon signal }=\int_{E_{\text {thresh }}}^{m_{\chi}} N_{\gamma}(E) \mathrm{d} E=8300 \text { photons } / \text { year } \alpha^{2} \int \mathrm{d} n_{\gamma}(E) \frac{<\sigma V>_{\text {halo }}}{10^{-26} \mathrm{~cm}^{3} / \mathrm{s}}\left(\frac{1 \mathrm{TeV}}{m_{\chi}}\right)^{2}
$$

The corresponding noise is the square root of the total number of background photons received above $E_{\text {thresh. }}$. From Equations 1, 20 and 3, it is approximately:

$$
\text { Soft photon noise }=\left\{\int_{E_{\text {thresh }}}^{m_{\chi}} N_{\text {backgr }}(E) \mathrm{d} E\right\}^{1 / 2} \simeq 220 \text { photons } / \sqrt{\text { year }} \frac{0.2 \mathrm{TeV}}{E_{\text {thresh }}}
$$

Note that we are conservative in integrating up to $m_{\chi}$ because the integral $\int \mathrm{d} n_{\gamma}(E)$ is saturated well before $m_{\chi}$, and therefore we are adding noise for no signal.

The signal to noise ratio will be very low below $m_{\chi} \simeq 1 \mathrm{TeV}$ unless annihilation cross-sections are large. But they cannot be very far from $<\sigma V>_{\text {halo }} \simeq 10^{-26} \mathrm{~cm}^{3} / \mathrm{s}$, if WIMP's constitute the dark matter of the universe. WIMP's were produced in large numbers during the hot dense phase of the BigBang, and to constitute the dark matter, some fraction must have survived annihilation up to now. This annihilation becomes negligible when the temperature gets lower than a critical decoupling temperature, and the present relic density $\Omega_{\chi}$ (in unit of the critical density) is 28 :

$$
\Omega_{\chi} h^{2}=\frac{2.5 \times 10^{-27} \mathrm{~cm}^{3} / \mathrm{s}}{<\sigma V>_{B B}}
$$

up to logarithmic corrections ( $h$ is the Hubble constant divided by $100 \mathrm{~km} / \mathrm{s} / \mathrm{Mpc}$, and we shall take $h=0.5)$. Note that the thermal average $\left\langle\sigma V>_{B B}\right.$ of the annihilation cross-section at the Big-Bang can differ from the thermal average $\langle\sigma V\rangle_{\text {halo }}$ in the halo, since the temperature at decoupling differs from the halo equivalent temperature.

To account for dark matter in galactic halos, a mean density $\Omega_{\text {halo }} \simeq 0.1$ is required [1, 2, 3, 何, while a mean density $\Omega \simeq 1$ seems required on larger scales. When the WIMP annihilation rate is strong, $\Omega_{\chi}<<1$, and the density of WIMP's in the halo cannot be much larger than $\rho_{\chi} \simeq \rho_{\text {halo }} \Omega_{\chi} / \Omega_{\text {halo }}$. The remaining component could be due to brown dwarfs [29] or to another WIMP. To interpolate the halo WIMP density between this strong annihilation rate and the weak annihilation rate (where $\rho_{\chi} \simeq \rho_{\text {halo }}$ ), Griest, Kamionkowski and Turner (GKT) [30] suggested the formula $\rho_{\chi} \simeq \rho_{\text {halo }} /\left[1+\Omega_{\text {halo }} / \Omega_{\chi}\right]$, but any smooth interpolation could be chosen. This GKT correction decreases the expected flux for a large annihilation cross-section, and Equation 8 becomes :

$$
\text { Soft photon signal } \simeq 8300 \text { photons } / \text { year } \alpha^{2} \int \mathrm{d} n_{\gamma}(E) \frac{<\sigma V>_{\text {halo }}}{10^{-26} \mathrm{~cm}^{3} / \mathrm{s}}\left(\frac{1 \mathrm{TeV}}{m_{\chi}}\right)^{2} \frac{1}{\left[1+\Omega_{\text {halo }} / \Omega_{\chi}\right]^{2}}
$$

\section{"Line" signal}

Two WIMP's can directly annihilate into two photons $\left(\int \mathrm{d} n_{\gamma}(E)=2\right)$, and the energy of the photons is then equal to the mass $m_{\chi}$ of the (non-relativistic) WIMP, leading to a gamma line. A narrow line in 
the range $10^{2 \pm 1} \mathrm{GeV}$ would provide a clear signature for dark matter annihilation [7, 8, 9, 10, 11, 12], but the cross-section $\sigma_{2 \gamma}$ for annihilation into 2 photons strongly depends on the WIMP model, and moreover it is expected to be much smaller than the annihilation cross-sections into a pair of quarks, leptons, Higgs or gauge bosons. For our reference telescope, the expected number of annihilation photons in the line at $E=m_{\chi}$ is :

$$
\text { Line signal }=166 \text { photons } / \text { year } \alpha^{2} \frac{<\sigma_{2 \gamma} V>_{\text {halo }}}{10^{-28} \mathrm{~cm}^{3} / \mathrm{s}}\left(\frac{1 \mathrm{TeV}}{m_{\chi}}\right)^{2} \frac{1}{\left[1+\Omega_{\text {halo }} / \Omega_{\chi}\right]^{2}}
$$

From Equation 1 , the background noise for such a $\gamma$ line, with an energy bin $\Delta E=3 \sigma=0.3 E$, is well approximated by :

$$
\text { Line noise }=\sqrt{N_{\text {backgr }}}=35 \text { photons } / \sqrt{\text { year }} \frac{1 \mathrm{TeV}}{m_{\chi}}
$$

Annihilations into a photon and another particle of mass $M$ also gives a monochromatic photon, at an energy $E \simeq m_{\chi}-M^{2} / 4 m_{\chi}$. Some kind of spectroscopy is thus in principle possible, depending on the energy resolution.

\section{Neutralinos}

We now focus on supersymmetry [14], the favorite extension of the standard model, because the lightest supersymmetric particle (LSP) $\chi$ is stable due to a conserved quantum number, R-parity, and because its relic density is naturally $\Omega_{\chi} \simeq 1$, making it a good dark matter candidate. There are two neutral gauge fermions $\tilde{\mathrm{B}}$ and $\tilde{\mathrm{W}}^{3}$ and two neutral Higgs fermions $\tilde{\mathrm{H}}^{1}$ and $\tilde{\mathrm{H}}^{2}$ which mix when gauge symmetry is broken, and the four resulting mass eigenstates are called neutralinos. The lightest one, the neutralino, usually is the LSP. Its mass and couplings depend on 4 parameters : the masses $M_{1}$ and $M_{2}$ of the gauge fermions $\tilde{\mathrm{B}}$ and $\tilde{\mathrm{W}}^{3}$ before gauge symmetry breaking (usually related by the GUT relation $M_{1}=\frac{5}{3} M_{2} \tan ^{2} \theta_{W}$ ), the Higgs fermion mass $\mu$ and the ratio $\tan \beta$ of the vacuum expectation values of the two Higgs scalars 14, 30, 31, 32, 33, 34. In addition, the neutralino cross-sections depend on the masses of the quarks and leptons, and of their scalar partners, and on the Higgs bosons masses. There are weak direct experimental lower bounds from LEP on the neutralino mass [35] but, assuming a GUT relation between the gluino mass $M_{3}$ and the masses $M_{1}$ and $M_{2}$, more stringent indirect bounds can be obtained from UA2 [36] and CDF 37] experiments. It is then likely that the mass of the neutralino is larger than about $50 \mathrm{GeV}$.

We are interested in the possibility that the neutralino be heavier than 100 to $200 \mathrm{GeV}$, the ACT threshold, because it allows a neutralino relic density near the critical density. We focus in this paper on the two extreme cases of a pure bino or a pure higgsino. A mixed state is not a priori excluded, but is quite unlikely in this high mass region : when $|\mu| \gg M_{2}$, the lightest neutralino is an almost pure $\tilde{\mathrm{B}}$ state, and an almost pure $\tilde{\mathrm{H}}$ when $|\mu| \ll M_{2}$. The equations for annihilation cross-sections are extremely complex [30, 31, 32, 33, 34, and we only give here a sketch of the results.

Most of the time, a bino pair annihilates into a quark or a lepton pair, through the exchange of the corresponding s-quark or s-lepton of mass $m_{\tilde{\mathrm{f}}}\left(m_{\tilde{\mathrm{f}}}>m_{\chi}\right.$ since, by assumption, $\chi$ is the LSP). The thermally averaged cross-section for binos at temperature $T$ is approximately :

$$
<\sigma V>=\frac{\pi \alpha^{2}}{216 \cos ^{4} \theta_{W}} \frac{m_{\chi}^{2}}{\left(m_{\tilde{\mathrm{f}}}^{2}+m_{\chi}^{2}\right)^{2}}\left\{289 \frac{m_{t o p}^{2}}{m_{\chi}^{2}}+9160 \frac{T}{m_{\chi}} \frac{m_{\tilde{\mathrm{f}}}^{4}+m_{\chi}^{4}}{\left(m_{\tilde{\mathrm{f}}}^{2}+m_{\chi}^{2}\right)^{2}}\right\}
$$

In this equation, $\alpha$ is the fine structure constant. As illustrated by Equation 14, thermally averaged cross-sections for neutralino annihilation can be written as $\langle\sigma V\rangle=a+b T / m_{\chi}$. Temperatures are very 
low in the galactic halo and the $a$ term dominates, whereas the $b$ term dominates during the Big-Bang, down to the decoupling temperature $T_{d e c} \simeq m_{\chi} / 25$. Equations 10 and 14 then yield a relation between the bino mass and its relic density $\Omega_{\chi}$ :

$$
m_{\chi}=0.56 \mathrm{TeV} h \sqrt{\Omega_{\chi}}
$$

This value was obtained assuming that $m_{\tilde{\mathrm{f}}}=m_{\chi}$, and it becomes much smaller if $m_{\tilde{\mathrm{f}}} \gg m_{\chi}$. Therefore cosmology leads to an upper bound on the bino mass 30, 31, 32, 33, 34]. It is very interesting that a similar upper bound is also indicated by naturalness arguments in the context of low energy supergravity models [38]. A negligibly small relic density is excluded for a bino by the experimental lower bounds on the neutralino mass and binos should be around us in large numbers, even if they do not solve the dark matter problem. Assuming again the lightest possible s-top mass, namely $m_{\tilde{\mathrm{f}}}=m_{\chi}$, the cross-section for annihilation in the halo into a top-antitop pair (for $m_{\chi}>m_{t o p}$ ) is :

$$
<\sigma V>_{\text {halo }} \simeq 2.8 \times 10^{-29} \mathrm{~cm}^{3} / \mathrm{s}\left(\frac{m_{\text {top }}}{150 \mathrm{GeV}}\right)^{2}\left(\frac{1 \mathrm{TeV}}{m_{\chi}}\right)^{4}
$$

if the bino is heavier than the top quark. The top quarks decay into a large number of soft photons, and protons, antiprotons, electrons, positrons, neutrinos and antineutrinos. The photon energy spectrum $\mathrm{d} n_{\gamma} / \mathrm{d} E$ is computed by Lund Monte-Carlo [27]. Figure 2 shows the expected excess in the number of photons in the direction of the center of the galaxy, seen by our reference telescope, as a function of the neutralino mass $m_{\chi}$. The excess number of photons is compared to the background noise measured in directions away from the center of the Galaxy. The bino annihilation into soft photons will be difficult to detect, unless the dark matter density enhancement at the galactic center is larger than we assumed (i.e. if $\alpha \gg 1$ ).

Let us now turn to a $\tilde{\mathrm{H}}$-like neutralino. The dominant higgsino annihilation channels are into WW or ZZ pairs, through the exchange of a chargino or a neutralino. The expression for its annihilation cross-sections is very complicated [30, 31, 32, 33], but, in the mass range we are interested in, it scales roughly as :

$$
<\sigma V>_{B B} \simeq 2.2 \times 10^{-26} \mathrm{~cm}^{3} / \mathrm{s}\left(\frac{1 \mathrm{TeV}}{m_{\chi}}\right)^{2}
$$

leading to :

$$
m_{\chi} \simeq 3 \mathrm{TeV} h \sqrt{\Omega_{\chi}}
$$

The bound is larger than for the bino, because higgsinos are more strongly coupled than binos. Moreover, at low temperature, the higgsino cross-section scales as $1 / m_{\chi}^{2}$ (fermion exchange) while the bino crosssection scales as $m_{\text {top }}^{2} / m_{\chi}^{4}$ (scalar exchange). In the halo, the cross-section for higgsino pair annihilation into W's and Z's is :

$$
<\sigma V>_{\text {halo }} \simeq \frac{\pi \alpha^{2}}{16} \frac{2 \cos ^{4} \theta_{W}+1}{\sin ^{4} \theta_{W} \cos ^{4} \theta_{W}} \frac{1}{m_{\chi}^{2}}
$$

Higgsino annihilation is larger than bino annihilation, both in the Big-Bang (hence a smaller relic density for a given mass) and in the halo (hence a larger photon flux). Figure 2 indeed shows that higgsino annihilation into soft photons would be easier to detect than bino annihilation. However the signal is still marginal for our reference telescope.

A gamma line would be a clearer signal. A neutralino pair cannot annihilate into two photons at the tree level, one loop graphs are required and probably lead to a tiny cross-section. There are many relevant graphs, in particular if annihilations into one photon and another particle ( $\mathrm{Z}^{0}$, Higgs scalar) are included. 
We are computing these graphs, but this long and tedious work is not yet completed. Nevertheless, an order of magnitude estimate may be derived from the graphs already computed [39]. For the annihilation of a bino into two photons through quark/s-quark or lepton/s-lepton loops, this leads to :

$$
<\sigma_{2 \gamma} V>_{\text {halo }} \simeq 4.2 \times 10^{-29} \mathrm{~cm}^{3} / \mathrm{s}\left(\frac{1 \mathrm{TeV}}{m_{\chi}}\right)^{2}\left(\frac{m_{\chi}}{m_{\tilde{\mathrm{f}}}}\right)^{4}
$$

Of course, cancellations may happen between graphs, and the cross-section of Equation 20 may be grossly overestimated. If it is not the case, Figure 3 shows that a bino could be detected at the 5 standard deviation level within one year of observation, down to the present $0.2 \mathrm{TeV}$ threshold, again assuming $m_{\tilde{\mathrm{f}}}=m_{\chi}$. The cross-section for the annihilation of a higgsino into two photons is not yet known, and we boldly assumed the same cross-section as for a bino. Figure 3 shows that the GKT correction factor suppresses the higgsino line signal, due to the smaller higgsino relic density for a given mass. Thus a higgsino would be more difficult to detect in this way. The two searches, for a soft photon excess and for a line signal, are therefore complementary, as they are sensitive to different types of neutralinos, and they can be done simultaneously within the same experiment.

\section{Conclusions}

Dark matter made of particles in the $100 \mathrm{GeV}-1 \mathrm{TeV}$ mass region could be detected through its annihilation products by an atmospheric Čerenkov telescope searching for energetic gamma rays. We apply this idea to the neutralino WIMP candidate from supersymmetry, and show that it may work, provided that :

i) the neutralino is heavier than about $200 \mathrm{GeV}$, which is one of the windows allowing a relic density $\Omega_{\chi}$ larger than $10^{-2}$.

ii) the density enhancement in the center of the Galaxy is as large as expected by the isothermal Ipser-Sikivie model.

iii) a large enough atmospheric Cerenkov detector is built in the southern hemisphere, and aimed towards the galactic center.

Improvements of the ACT technique to lower the energy threshold are of paramount importance to close the gap between the $0.05 \mathrm{TeV}$ or so of present accelerator experiments, and the $0.2 \mathrm{TeV}$ expected for our reference telescope. A complementary study could be based on the detection of the charged decay products, the electrons, protons and antiprotons. There is no directionality any more, and so no on-off experiment is possible, but consequently there is no prefered location for the apparatus. Integrating all over the galactic halo, preliminary calculations suggest that antiprotons (and protons) must display a strong enhancement below the neutralino mass, while other nuclei should not.

\section{References}

[1] S.M. Faber and J.S. Gallagher, Ann. Rev. Astron. Astrophys. 17 (1979) 135

[2] V. Trimble, Ann. Rev. Astron. Astrophys. 25(1987)425

[3] J. Kormandy and G.R. Knapp (eds), Proceedings of the IAU Symposium 117, "Dark matter in the universe" (Reidel 1987) 
[4] M.S. Turner," Dark matter in the universe", talk at Nobel Symposium 79 "The birth and early evolution of our universe" (Graftavallen, 11-16 June 1990) Fermilab-Conf 90/230A

[5] J. Primack, D. Seckel and B. Sadoulet, Ann Rev. Nucl. Part. Phys. 38(1988)751

[6] P.F. Smith and J.D. Lewin, in 3rd ESO-CERN Symposium, M. Caffo et al. eds (Kluwer, 1989)

[7] J.E. Gunn et al., Ap.J. 223 (1978) 1015

[8] F.W. Stecker, Ap.J. 223(1978)1032

[9] J.Silk and M. Srednicki, Phys. Rev. Lett. 53 (1984) 624

[10] L. Bergström and H. Snellman, Phys. Rev. D37 (1988) 3737

[11] S. Rudaz, Phys. Rev. D39 (1989) 3549

[12] A. Bouquet, P. Salati and J. Silk, Phys. Rev. D40 (1989) 3168

[13] T.C. Weekes, Phys. Rep. 160 (1988) 1

[14] H.P. Nilles, Phys. Rep. 110 (1984) 1

[15] J.Nishimura et al., Ap.J. 238 (1980) 394

[16] T.D.He and Q.Q. Bhu, Phys.Rev. D44 (1991) 2635.

[17] H.A. Mayer-Hasselwander et al., Astr. and Astroph. 105 (1982) 164

[18] Chaman L.Bhat et al., Nature 314 (1985) 511

[19] H.Bloemen, Ann.Rev.Astr.Astroph. 27 (1989)

[20] J. N. Bahcall and R.M. Soneira, Astrophys. J. Supp. 44 (1980) 73

[21] J.A.R. Caldwell and J. Ostriker, Ap. J. 251 (1981) 61

[22] J. Binney and S. Tremaine, "Galactic Dynamics" (Princeton University Press, Princeton, 1987)

[23] R.A. Flores, Phys. Lett.B 215 (1988) 73

[24] Ya.B. Zel'dovich et al, Sov. J. Nucl. Phys. 31 (1980) 664

[25] D.P. Clemens, Ap. J. 295 (1985) 422

[26] J.R. Ipser and P. Sikivie, Phys. Rev. D35 (1987) 3695

[27] H.U. Bengtsson and T. Sjostrand, Computer Phys. Comm. 46 (1987) 43

[28] E.W. Kolb and M.S. Turner, "The early universe", (Addison-Wesley, New-York 1990)

[29] M.J. Rees, "Baryonic dark matter", 2nd ESO-CERN Symposium (1986)

[30] K.Griest, M.Kamionkowski and M.S.Turner, Phys. Rev. D41 (1990) 3565

[31] J.Ellis et al., Nucl. Phys. B238 (1984) 453

[32] H.E. Haber and G.L. Kane, Phys. Rep. 117 (1985) 75 
[33] K. Griest, Phys. Rev. D38 (1988) 2357

[34] K.A. Olive and M. Srednicki, Phys. Lett. B230 (1989) 78

[35] D. Decamps et al., ALEPH Collaboration, Phys. Lett. 244B (1990) 541

[36] J. Alitti et al., UA2 Collaboration, Phys. Lett. 235B (1990) 363

[37] F. Abe et al., CDF Collaboration, Phiys. Rev. Lett. 62 (1989) 1825ys. Rev. Lett. 62 (1989) 1825

[38] R. Barbieri and G.F. Giudice, Nucl. Phys. B306 (1988) 63 o: hep-ph@xxx.lanl.gov Subject: put

[39] G.F. Giudice and K. Griest, Phys.Rev. D40 (1989) 2549

\section{FIGURE CAPTIONS}

Figure 1 : Photon energy spectrum from the pair annihilation of neutralinos, showing the continuum due to quarks, leptons, Higgs and gauge boson decays, computed through a Lund Monte-Carlo, and the lines (convolved with experimental energy resolution) due to direct annihilations into two photons, and a heavy particle and a photon. The relative size of the lines and of the continuum is arbitrary.

Figure 2 : Soft photon signal. Expected excess number of events per year above a $100 \mathrm{GeV}$ threshold for a bino or a higgsino-like neutralino, as a function of the neutralino mass $m_{\chi}$, compared to the noise (one-sigma fluctuations of the background). The bino and higgsino curves correspond to an Ipser-Sikivie model of the halo core with $\alpha=1$, using the GKT correction to reduce the flux when the neutralino relic density $\Omega_{\chi}$ is smaller than the halo mean density $\Omega_{\text {halo }}=0.1$, and assuming a s-fermion mass $m_{\tilde{\mathrm{f}}}=m_{\chi}$. The two vertical lines correspond to $\Omega_{\chi} h^{2}=1$ for a bino and for a higgsino.

Figure 3 : Line signal. Expected number of events per year in the line for a bino or higgsino-like neutralino, as a function of the neutralino mass $m_{\chi}$, compared to the noise (one-sigma fluctuations of the background). The bino and higgsino curves correspond to an Ipser-Sikivie model of the halo core with $\alpha=1$, using the GKT correction, and assuming a s-fermion mass $m_{\tilde{\mathrm{f}}}=m_{\chi}$, and an energy resolution $\Delta E=0.3 E$. The two vertical lines correspond to $\Omega_{\chi} h^{2}=1$ for a bino and for a higgsino. 